Ueber die ungleiche Vertheilung der Warme auf der Sonnenoberfläche.

In den Comptes rendus der Pariser Akademie hat Herr Prof. Seccli vor Kurzem mehrlache Mittheilungen von thermoelektrischen Messungen auf der Sonnenscheibe gemacht, aus denen bervorzugehen scheint, dass die Wärme vom Sonuenäquator nach den Polen hin merklich abnimmt. Da dieser Messungen in den Astr. Nachr. (Bd. XXXV. S. 277) bereits Erwähruung geschehen ist, so erlaube ich mir hier auszugsweise die Re. sultate aus einer Rechnung anzufïhren, die ich auf Grund der Königsberger und Berliner Temperaturbenhacltungen über die ungleiche Wärmevertheilung nach den $L$ ängengraden der Sonne angestellt halse, und die also in eimigem $\mathbf{Z u}$ sammenhauge mit jeusen Messungen steht. Erinnern nämlich Secchi's Resultate an die bereits im Jahre 1845 von Herm Prof. Nervander in Helsingfors nachgewiesene Temperaturgleichung, deren Periode mit der Umdrehungszeit der Sonae zusammenfällt, so wird man doch nicht übersehen, dass das wirkliche Vorhaudensein einer solchen Gleichung hisher nur an zwei oder drei Orten nachgewiesen wurde, während dieselbe erst einige Wahrscheinlichkeit gewinnen wird, wenn sich unter sehr verschiedenen klimatischen Verhältnissen der hier eintretende Coefficient nahezu von derselhen Grösse ergeben sollte.

Ausser jener ersten Untersuchung von Nervander, welche sich auf Pariser und Insprucker Beobachtungeu stützt *) ist nur noch eine hier zu vergleichende Arbeit von Carlini bekannt geworden, weicher ans den Mailänder Beobachtungen die von der Rotation der Sonne herrührende grösste Ungleichheit fast in vollkonmener Cebereinstimnung findet mit den Ergebuissen aus Paris und luspruck $*$ ). Theilt man nämlich, um von der täglichen Periude frei zu sein, beispielsweise die Mittagstemperatur-Beobachtungen vicler Jahre dergestalt in Gruppen, dass man alle die Ablesungen in eine Summe vereinigt, welche der Rückkehr eines festen Sonnenmeridianes in eine bestimmte Lage zur Erde entsprechen, so wird sich, wenn die vorausgesetzte Ungleichheit üherhaupt vorbanden ist, der grösste Unterschied durch Interpolation soggleich ergeben. Auf diese Weise hat man gegenwärtig, aus dell angeführten Quellen, zur Vergleichung nur folgende

Unterschiede zwischen Maximum und Minimum:

Insprucker Beobb., 1777-1828....0 $0^{\circ} 600 \mathrm{C}$. Nervander.

Pariser ", 1816-1839...0,604,

Mailänder $" \quad 1835-1844 \ldots . .0,712, "$ Carlini.

") Bulletins de la classe ph. math. de l'Académie de St. Pétersbourg. T. III. No. 49.

**) Giornale dell' J. R. Istituto Lombardo cet. T. VI. p. 448. (Milano 1846).
Aus einigen andern Arbeiten, welche seitdem über denselhen Gegenstand bekannt gewordeu siud, kann man eine so einfache, directe Vergleichung nicht entnehmen, welche üher das Vorhandensein der Ursache erst entscheiden unss, bevor Folgerungen daraus zulässir erscheinen kümnen.

Bei der Zusammenstellung der auf der $\mathbf{K}$ änigsberger Sternwarte von 1827-1837 im Miftag heobachteten Temperaturen bin ich genau dem Carlini"schen Verfahren gefolgt. Indem ich von denijenigen Sonnenmeridian $\boldsymbol{M}$ ausging, der am

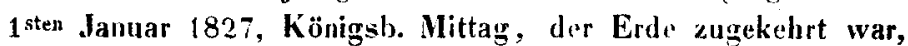
habe ich, unter Zugrundelegung der geocentrischen Umdrehungszeit der Sonne von $27,26 \mathrm{~m}$. Tage. in der ersten Gruppe die Summen aller Temperaturbeobachtungen gebildet, welche zu diesen Meridiane gebören. Die zwrite Gruppe zeigt die Summen, welche zum Läugengrade der Sonne $M+120^{\circ}$ gehören; die dritte die dem Meridian $M+240^{\circ}$ entsprechenden Summen. Der Umstand, dass die Zeiten, in welchen der betreffende Meridian der Erde zugekehrt ist, nur selten in den Mittag selbst fallen, wurde dadurch unschädlich gemacht, dass his zu $6^{\text {h }}$ hin und von $18^{\text {th }}$ an stets der nächstliegende Mittag (l), zwischen $6^{\text {b }}$ und $18^{\text {h }}$ aber der vor- und der nachgehende Mittag (II. und 1II.) angesetzt wurden. Dadurch entstanden folgende Tafelo:

Erste Grupue. Meridian $M$.

\begin{tabular}{|c|c|c|c|c|c|c|}
\hline Jahr. & II. & III. & $\frac{1}{2}($ II. + III.) & I. & Susmme. & Beobu \\
\hline 1827 & 336,7 & 329,8 & 333,25 & 339,70 & 672,95 & 14 \\
\hline 1828 & 261,4 & 287,4 & 274,40 & 330,90 & 605,30 & 14 \\
\hline 1829 & 309,9 & 303,7 & 306,80 & 292,50 & $\dot{5} 99,30$ & 14 \\
\hline 1830 & 339,3 & 333,0 & 336,15 & 277,20 & 613,35 & 13 \\
\hline $1832 *)$ & 328,8 & 313,3 & 321,05 & 284,70 & 605,75 & 13 \\
\hline 1833 & 376,2 & 374,3 & 375,25 & 253,10 & $628,3 \overline{5}$ & 13 \\
\hline 1834 & 381,6 & 403,5 & 393,55 & 298,10 & 691,65 & 14 \\
\hline 1835 & 339,4 & 326,0 & 332,70 & 334,70 & 667,40 & 13 \\
\hline 1836 & 307,1 & 315,6 & 311,35 & 327,20 & 638,55 & 13 \\
\hline 1837 & 283,9 & 278,6 & 281,25 & 328,70 & 609,95 & 14 \\
\hline
\end{tabular}

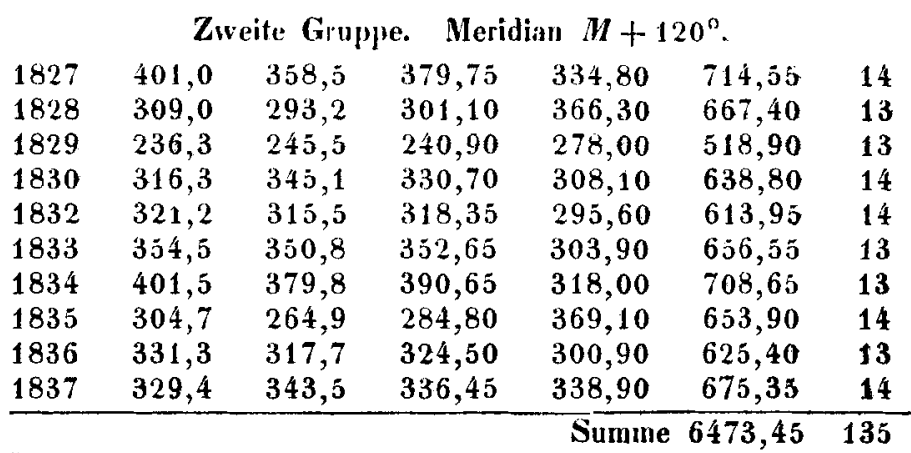

*) 1831 wurden alle Beobb. zwei Monate hindurch augesetzt. 
Dritte Gruppe. Meridian $M+240^{\circ}$.

\begin{tabular}{|c|c|c|c|c|c|c|}
\hline Jahr. & II. & III. & $\frac{1}{2}(I I+I I I)$. & I. & Summe. & Beols. \\
\hline 1827 & 271,7 & 264,6 & 268,15 & 354,70 & 622,85 & 13 \\
\hline 1828 & 305,4 & 293,9 & 299,65 & 336,40 & 636,05 & 13 \\
\hline 1829 & 309,6 & 322,9 & 316,25 & 268,70 & 584,95 & 14 \\
\hline 1830 & 285,9 & 315,3 & 300,60 & 299,60 & 600,20 & 13 \\
\hline 1832 & 332,0 & 323,7 & 327,85 & 277,10 & 604,95 & 13 \\
\hline 1833 & 428,3 & 433,6 & $430,9 j$ & 249,80 & 680,75 & 14 \\
\hline 1834 & 400,7 & 398,2 & 399,45 & 322,30 & 721,75 & $\$ 3$ \\
\hline 1835 & 338,8 & 335,7 & 337,25 & 286,70 & 623,95 & 13 \\
\hline 1836 & 353,1 & 372,0 & 362,55 & 314,30 & 676,85 & 14 \\
\hline 1837 & $284, \pi$ & 275,3 & 280,00 & 311,30 & 591,30 & 13 \\
\hline
\end{tabular}

Ist mun $t$ die nittlere Mittagstemperatur für Königsberg, $\theta$ der Coeflicient der Ungleichheit, welche von der Sonnenrotation herrührt, $N$ eiue Constante und $m$ der Winkel, welchen irgend ein der Erde zugewandter Sonnenmeridian mit den festen Meridian $\boldsymbol{M}$ bildet, so wird die $z \mathfrak{u} m$ gebörige Temperatur $t^{\prime}$ ausgedrückt durch

$$
t^{\prime}=t+\theta \sin (N+m)
$$

und es bestehen also nach Obigem, in Fahrenheit'schen Graden, die folgenden drei Gleichungen:

$$
\begin{aligned}
& 46,908=t+\theta \sin N \\
& 47,931=t-\frac{1}{2} \theta \sin N+\frac{1}{2} \theta \cos N \cdot \sqrt{3} \\
& 47,696=t-\frac{1}{2} \theta \sin N-\frac{1}{2} \theta \cos N \cdot V 3 .
\end{aligned}
$$

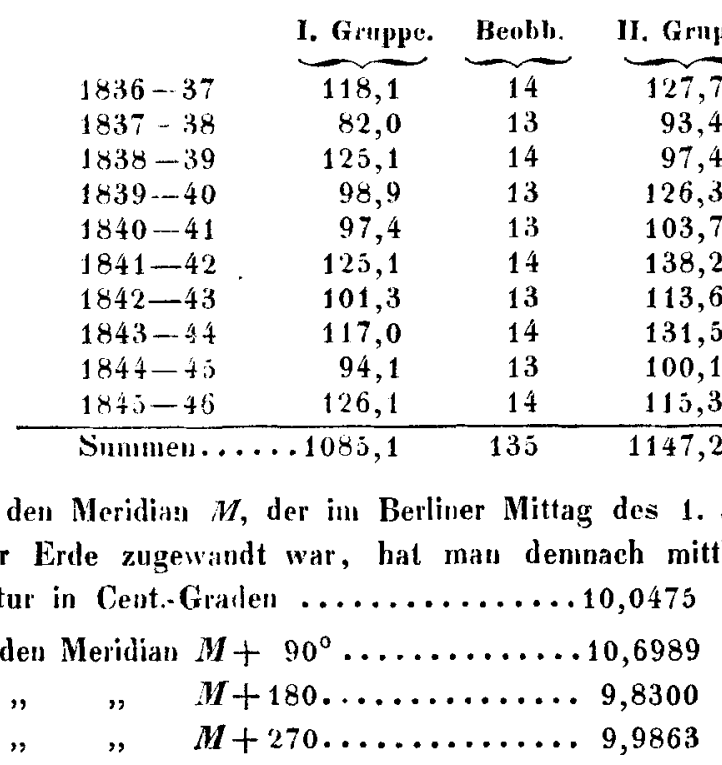

Man sieht nun bald, dass diese Werthe auf eine beträchtlich grössere Ungleichheit führen würden, und dass sie keineswegs durch die oben für Königsberg gewählte Form in annehmbarer Weise darzustellen siud. In gegenwärtigem Falle wird etwa

$$
\begin{aligned}
\text { Mittl. Temp. }=10^{\circ} 1406 & +0^{\circ} 3724 \sin \left(16^{\circ} 59^{\prime}+m\right) \\
& -0^{\circ} 3615 \sin \left(33^{\circ} 57^{\prime}+2 m\right),
\end{aligned}
$$

1836 ler Erde zugewandt war, hat man dennach mittlere Temperatur in Cent.-Gralen ............ 10,0475

$$
\begin{array}{ccc}
\text { für den Meridian } M+90^{\circ} \ldots \ldots \ldots \ldots \ldots \ldots 10,6989 \\
, ", \quad, \quad M+180 \ldots \ldots \ldots \ldots \ldots \ldots 9,8300
\end{array}
$$

Danach wird in Centesimalgraden der Ausuluck fïr die Mittager temperatur von Königsberg seiu

$$
+8^{\circ} 621+0^{n} 3487 \sin \left(283^{\circ} 37^{\prime}+m\right)
$$

wo $m$ in 27,26 Tagen den Umkreis durchläult, indess ist somit der von der ungleichen Wärmevertheilung auf der Sonne herrïhrende grösste Unterschicd der Temperaturen fast gena der von Carlini gefundene, nämlich $0^{\circ} 697$ statt des Mailänder $0^{\circ} 712 \mathrm{C}$.

Diese frappante Uebereinstimmung der Resultate verräth sich indessen, wenn man nach weiterer Bestätigung sucht,

\begin{tabular}{|c|c|c|c|c|}
\hline Beobb. & III. Gruppe. & Beobh. & IV. Gruppe. & Bcobb. \\
\hline 14 & 82,0 & 13 & 87,2 & 13 \\
\hline 13 & 86,6 & 14 & 112,9 & 14 \\
\hline 13 & 95,6 & 13 & 93,5 & 13 \\
\hline 14 & 108,3 & 14 & 112,5 & 13 \\
\hline 13 & 98,8 & 13 & 116,4 & 14 \\
\hline 14 & 111,4 & 13 & 110,1 & 13 \\
\hline 13 & 132,5 & 14 & 116,1 & 14 \\
\hline 13 & 107,0 & 13 & 100,9 & 13 \\
\hline 14 & 98,9 & 14 & 89,2 & 13 \\
\hline 13 & 132,7 & 13 & 131,8 & 14 \\
\hline 134 & 1053,8 & 134 & 1070,6 & 134 \\
\hline
\end{tabular}
uur als eine zufällige, theilweise wohl herbeigeführt durch die ganz gleiche Behandlungsweise der Beobachtungen. Nach dem ersten Erfolge fast zu der Erwartung berecbtigt, dieselbe Grösse der Ungleichbeit auch bei veränderter Anordnung aus Professor Galle's Berliner Beobachtungen herleiten zu können, legte ich, diesmal von Juli 1. 1836 ausgehend, vier auf einander senkrechte Meridiane über die Sonne. Dann stehen in Réaumur'schen Graden die Summen für die einzeluen Jahre so : wo $m$ wiederum innerhalh 27,26 Tagen den Umkreis durchläuft. Die grössten und kleinsten Werthe ergeben sich aus

$$
\cos \left(16^{\circ} 59^{\prime}+m\right)=0,12753 \pm 0,71850
$$

und werden somit

$$
\begin{aligned}
& \text { erstes Minimum } \ldots 10^{\circ} 013 \mathrm{C} \text {. } \\
& \text { absol. Maximum } \ldots 10,786 \\
& \text { absol. Minimum } \ldots 9,495 \\
& \text { aweites Maximum } \ldots 10,268
\end{aligned}
$$

Der grösste Unterschied ist nun nahe auf das Doppelte gestiegen, und wenn auch aus den bisher untersuchten Beobachtungen allerdings das Vorhandensein einer von der Umdrehung der Sonne abhängigen Variation, und zwar, wie es jetzt scheint, 
einer Variation ohne gleichmässige Zu- und Abrahme, gefolgert werden dürfte, so wird man doch den wahren Betray derselben aus Temperatur-Beobachtungen schwerlich berleiten können. Das Vorhandensein von wenigstens zwei grössten und kleinsten Werthen vermuthete schon Nervander (Bull. III. p. 14); fände es aus weiteren Rechnungen oder hesser aus directen Messungen, wie die von Secchi für die Breitengrade der Sonne angestellten, Bestätigung, so würde man sich kaum enthalten können, an eine Analogie mit den Lichterscbeinungen zu denken, welche $\beta$ Lyrae nach Argelander bietet.

Leipzig, im Octoher 1853.

\section{H. d'Arrest.}

Beobachtungen auf der Senfienberger Sternwarte.

Comet von Westphal.

M. Zt. Senftenb.

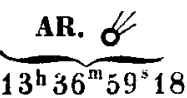
$\underbrace{\text { Decl. of }}_{+79^{\circ} 38^{\prime} 12^{\prime \prime} 8} \underbrace{\text { Vergl. }}_{8}$

18520

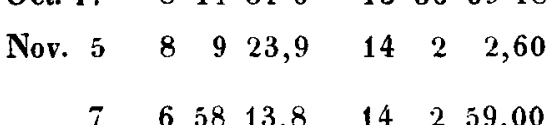

$\begin{array}{lll}70 & 27 & 57,4\end{array}$

$12\}$

695139,8 $13^{\mathrm{h}} 42^{\mathrm{m}} 2^{\mathrm{s}} 16+79^{\prime \prime} 36^{\prime} 45^{\prime \prime} 8$

14916,53

$\begin{array}{lll}14 & 8 & 10,79\end{array}$

10\{
70732,0

$\begin{array}{llll}70 & 45 & 22,1\end{array}$

$\begin{array}{rrrrrr}14 & 7 & 8,55 & 69 & 33 & 28,9 \\ 14 & 9 & 16,69 & +70 & 7 & 31,4\end{array}$
Arg. Z. 194 No. 10.

- 115, 204; Radcl. I.X.

Radcl, 4, 6, 9 .

Comet von Klinkerfues.

1853 Aug. $26 \quad 75248$,

$1135 \quad 14,48$
$+211650,67113949,87+205953,6$

B. N. 467 .

\begin{tabular}{|c|c|c|c|c|}
\hline 53 März 3 & 3 & 9 & 59 & 6,0 \\
\hline & 5 & 7 & 52 & 28,2 \\
\hline 10 & 0 & 7 & 58 & 1 \\
\hline 11 & 1 & 9 & 6 & 50 \\
\hline 12 & 2 & 9 & 7 & 17 \\
\hline 26 & 6 & 8 & 22 & 48 \\
\hline 28 & 8 & 8 & 48 & 19 \\
\hline & 9 & 8 & 40 & 41 \\
\hline April 1 & & 8 & 54 & 1 \\
\hline 11 & 1 & 9 & 26 & 29 \\
\hline & 25 & 9 & 13 & \\
\hline
\end{tabular}

x. Plan.

$358 \quad 36,05$

$\begin{array}{lll}4 & 1 & 46,99\end{array}$

$4 \quad 1025,22$

$412 \quad 17,10$

$\begin{array}{lll}4 & 14 & 5,62\end{array}$

$4 \quad 40 \quad 55,55$

4453,84

$4 \quad 47 \quad 6,83$

$\begin{array}{lll}4 & 53 & 24,28\end{array}$

$\begin{array}{lll}5 & 15 & 10,47\end{array}$

$5 \quad 4720,80$

Tha lia.

d. Planet. $+244532,4 \quad 6$

$2458 \quad 41,9$

$\begin{array}{lll}25 & 32 & 37,8\end{array}$

$\begin{array}{lll}25 & 39 & 31,3\end{array}$

$25 \quad 46 \quad 0,5$

$27 \quad 10 \quad 24,9$

$2721 \quad 14,7$

$27 \quad 26 \quad 21,6$

$\begin{array}{lll}27 & 41 & 39,7\end{array}$

$\begin{array}{lll}28 & 25 & 11,5\end{array}$ 285
$+29547,2$

6
22
10
10
10
6
21
9
5
20
33

\section{$348 \quad 20,95$}

4142,26

$\begin{array}{lll}413 & 37,66\end{array}$

$\begin{array}{lll}4 & 16 & 12,01\end{array}$

41133,19

44828,68

44335,48

$+5025,55$

$\begin{array}{lll}4 & 43 & 35,41\end{array}$

$\begin{array}{lll}5 & 16 & 59,00\end{array}$

$\begin{array}{llll}5 & 47 & 12,33\end{array}$

$\begin{array}{rrr}+24 & 51 & 0,8 \\ 24 & 58 & 19,3 \\ 25 & 16 & 40,5 \\ 25 & 24 & 21,2 \\ 25 & 28 & 4,6 \\ 27 & 8 & 47,2 \\ 27 & 38 & 49,1 \\ 27 & 29 & 6,6 \\ 27 & 38 & 48,9 \\ 28 & 28 & 43,3 \\ +28 & 54 & 51,1\end{array}$

Rümker 1038.

B. Z. 395 .

B. A. C.

B. Z. 396 .

- 396 .

396,397 .

b. A. C. 1497 , Rümk. 1303 .

B. Z. 397 .

B. A.C. 1497 .

$\beta$ Tauri.

B.A.C. 1882 .

C a 11 i o pe.
1853 März $5 \quad 9 \quad 28 \quad 22,6$
$44628,52+29455 j, 7$
$45256,90+294639,6$
B. Z. 398 .
$\begin{array}{llll}10 & 9 & 37 & 54,9\end{array}$
4522,3
$29 \quad 58 \quad 33,0$
$448 \quad 6,25$
$\begin{array}{lll}29 & 56 & 19,5\end{array}$

Der in B. Z. 39\% als Doppelsteru 8. Gr. aufgefïhrte Vergleichstern zo T'halia, März 29, kommt jetzt am Himmel nur einfach vor. Der scheinbare ort ist nach Vergleichungen mit einem andern Stern dieser Zome aus dem vorangehend beobachteten reducirt worden.

Beobachtungen der Hebe am Königsberger Reichenbach'schen Meridiankreise.

\begin{tabular}{|c|c|c|c|c|c|c|}
\hline \multirow{3}{*}{1853 Febr. } & & M. Zt. Königsb. & Sch. AR. & Sch. Decl. & Ephem. & Beol. \\
\hline & 1 & $11^{\mathrm{h}} 7^{\mathrm{m}} 16^{\mathrm{s}} 4$ & $7^{\mathrm{h}} 55^{\mathrm{m}} 17^{\mathrm{s}} 96$ & $+14^{\circ} 28^{\prime} 43^{\prime \prime} 8$ & $-0 \times 37$ & $-3^{\prime \prime} 4$ \\
\hline & 2 & 227,4 & $54 \quad 24,78$ & $38 \quad 15,9$ & $-0,23$ & $-3,3$ \\
\hline & 3 & 105740,1 & 75333,02 & $+144744,6$ & $-0,44$ & $-2,9$ \\
\hline
\end{tabular}

Die Vergleichung bezieht sich auf Hertn Luther's Ephemeride im Berliner Jahrbuche. Der Planet erschien 9. Grösse. A. Marth. 